\title{
Intermediate-Mass Black Hole Candidate ULXs
}

\author{
J. Miller \\ Harvard-Smithsonian Center for Astrophysics, Cambridge, Mass., USA \\ email: jmmiller@cfa.harvard.edu
}

\begin{abstract}
In a subset of the most luminous of the so-called "ultra-luminous" X-ray sources in nearby galaxies, there is evidence for black holes with masses considerably higher than found in Galactic binaries. Apart from extremely high X-ray luminosities, cool disks found in the X-ray spectra of these sources and X-ray timing measurements form the basis for present evidence for intermediate-mass black holes in these sources. New optical and radio measurements appear to support the X-ray evidence. I will review recent X-ray, optical, and radio observations of these ULXs, and discuss the strengths of the intermediate-mass black hole interpretation, arguments against this interpretation, and future prospects for revealing the nature of these ULXS more clearly.
\end{abstract}

\section{Discussion}

Ghosh: Regarding diagnostics from PDS, you described QPOs at some length. What about the possible diagnostic value of the "break" in PDS? Can you comment on the status of our understanding?

MILLER: The nature of breaks is not well-known. Breaks may trace a timescale which is fundamental (see correlation between stellar mass BHs and AGN). But, the modelling of PDS is ambiguous. Spectra are a better route to understanding ULXs.

MACCARONE: Could you comment on the fact that the spectral index of the power law is, for example for the NGC 1313 X-1 ULX, much harder than what is typically seen in Galactic very high state systems, but similar to low/hard states? Could this imply even higher masses than you've suggested?

MILLER: I think these might be similar to GX339-4 at its peak, where it's in the low/hard state, but very bright. 